\title{
Tailorable aqueous-media synthesis of heterogeneous Copper nanoparticles hybrids and application in selective oxidation of benzene
}

\author{
Noelia Losada-Garcia ${ }^{\mathrm{a}}$, Alba Rodriguez-Otero ${ }^{\mathrm{a}}$ and Jose M. Palomo ${ }^{\mathrm{a} *}$
}

${ }^{\text {a }}$ Department of Biocatalysis. Institute of Catalysis (CSIC). Marie Curie 2.

Cantoblanco. Campus UAM, 28049 Madrid, Spain. Fax: +34-91-585-4760.

E-mail: josempalomo@icp.csic.es 


\begin{abstract}
Novel heterogeneous nanocatalysts has been synthesized in aqueous media at multimilligram scale for highly selective direct oxidation of benzene to phenol in aqueous media. The synthesis of a novel biohybrids containing copper nanoparticles (CuNPs) by an efficient and green technology have been described. The methodology involves the combination of an enzyme and a copper salt in aqueous media at room temperature. It was possible to control the copper species and nanoparticle size depending on the experimental conditions, e.g. $\mathrm{pH}$, reducing step, amount of enzyme, obtaining novel heterogeneous nanobiohybrids containing exclusively $\mathrm{Cu}(0) \mathrm{NPs}, \mathrm{Cu}_{2} \mathrm{O}$ $(\mathrm{Cu}(\mathrm{i}))$ NPs or very crystalline $\mathrm{Cu}_{3}\left(\mathrm{PO}_{4}\right)_{2}(\mathrm{Cu}$ (ii)) NPs. Very small dispersed copper nanoparticles were formed in all cases (from 3 to $15 \mathrm{~nm}$ ). These novel CuNPs biohybrids were evaluated as catalyst in the selective oxidation of benzene to phenol in water at $30^{\circ} \mathrm{C}$ using hydrogen peroxide as oxidant, obtaining excellent yields and selectivity of phenol (>80\% yield, $>95 \%$ selectivity).
\end{abstract}




\section{Introduction}

Copper is an earth-abundant and low cost metal, which has been described very useful for different applications, such as antimicrobial agent ${ }^{1-2}$, environmental remediation ${ }^{3-4}$, electronics ${ }^{5}$ and specially in catalysis. ${ }^{6-9}$ Indeed, it has been a very extensive work in the recent years in the area of application of $\mathrm{Cu}$ catalysts in chemical reactions, especially in $\mathrm{C}-\mathrm{C}$ bond (click chemistry, $\mathrm{C}-\mathrm{H}$ activation, etc.), selective oxidation and reductions. ${ }^{7-}$ 9

In particular, $\mathrm{Cu}$ nanoparticles ( $\mathrm{Cu} \mathrm{NPs}$ ) have generated a great deal of interest in recent years. ${ }^{10}$ The high surface-to-volume ratio of nanomaterials compared to bulk materials generally makes them attractive candidates for their application as catalysts. ${ }^{11}$

Actually, it has been described different practical and straightforward multiple ways of preparing $\mathrm{Cu}$ nanomaterials. ${ }^{12-14}$ However, the use of $\mathrm{Cu}$ NPs is restricted by copper inherent instability under atmospheric conditions, which makes it prone to oxidation. Many efforts to develop the methods and supporting materials that increase the stability of $\mathrm{Cu}$ NPs by altering their sensitivity to oxygen, water and other chemical entities has encouraged the exploration of alternative $\mathrm{Cu}$-based NPs with more complex structures, such as core/shell Cu NPs or systems based on copper oxides. ${ }^{15-20}$

The synthesis of $\mathrm{Cu}$ and copper oxide NPs essentially centres around mainly four chemical reaction types, reduction, oxidation, hydrolysis or condensation. Depending on the choice of final materials, either one or a combination of aforementioned chemistries can be applied. The synthesis of $\mathrm{Cu}$ NPs often entails the reduction of $\mathrm{Cu}$ (i) or $\mathrm{Cu}$ (ii) sources. The synthesis of copper oxide NPs, on the other hand, basically requires hydrolysis of the precursors followed by a dehydration process leading to the final materials. Additionally, an oxidation process (sometimes unavoidable for $\mathrm{Cu}$-based NPs) can be deployed for the preparation of $\mathrm{Cu}$ based NPs with higher oxidation 
numbers from their respective precursors of lower oxidation states. In synthetic processes, the techniques that are applied provide a suitable environment and energy to facilitate the process of choice while additional constraints are imposed to modulate the stability, properties and morphology of the final NPs. ${ }^{21-23}$

Therefore, the development of methodologies to preparing highly active, selective, stable and robust copper nanoparticles is mandatory. ${ }^{24}$ Also sustainable and inexpensive systems that permitting high amount of CuNPs is also desirable from a more applicable point of view.

The copper species, in term of catalysis, are one of the important aspects on the final properties of the nanocatalysts in a particular reaction. Therefore, the development of technologies which permit to control the synthesis specifically of one copper specie represents an important challenge.

Selective catalytic oxidation of inactive aromatic $\mathrm{C}-\mathrm{H}$ bonds is one of the most active research topics, but remains a long-term challenge. ${ }^{25}$ Phenol is the most important industrial chemical due to its wide application in the fields of phenolic resin, pharmaceutical products, agrochemicals, etc. ${ }^{26}$ However, the current industrial production of phenol is still limited to the three-step cumene process, which includes propylene benzene, autoxidation to cumene hydroperoxide and rearrangement of Hock (Scheme 1). ${ }^{16}$ These reactions are carried out at elevated temperatures, high pressure and in extremely acidic conditions, resulting in a low overall phenol yield $(<5 \%)$ and an equimolar amount of acetone-derived product. ${ }^{27}$ Therefore, the direct catalytic oxidation in one step from benzene to phenol with peroxide hydrogen on heterogeneous catalysts remains a central theme of fundamental and applied research. Recently, different strategies have been developed for the more sustainable production of this valuable "building block" by means of oxidative methods or the use of photocatalysis although 
most of cases low or moderate yields and non-total selectivity has been obtained, ${ }^{26,28-30}$ being reported only a few recent examples with higher conversion degree..$^{31-33}$ In this work, a process to producing in situ Controlled-Cu species and nanoparticle size in a protein host network of $\mathrm{Cu}$ hybrids materials has been described for the first time and demonstrated by X-ray diffraction (XRD) and transmission electron microscopy (TEM).

Furthermore, some of these new $\mathrm{Cu}$ nanobiohybrids showed excellent results as economical heterogeneous catalysts in the direct sustainable selective conversion of benzene in phenol in aqueous media at $30^{\circ} \mathrm{C}$ (Scheme 1).

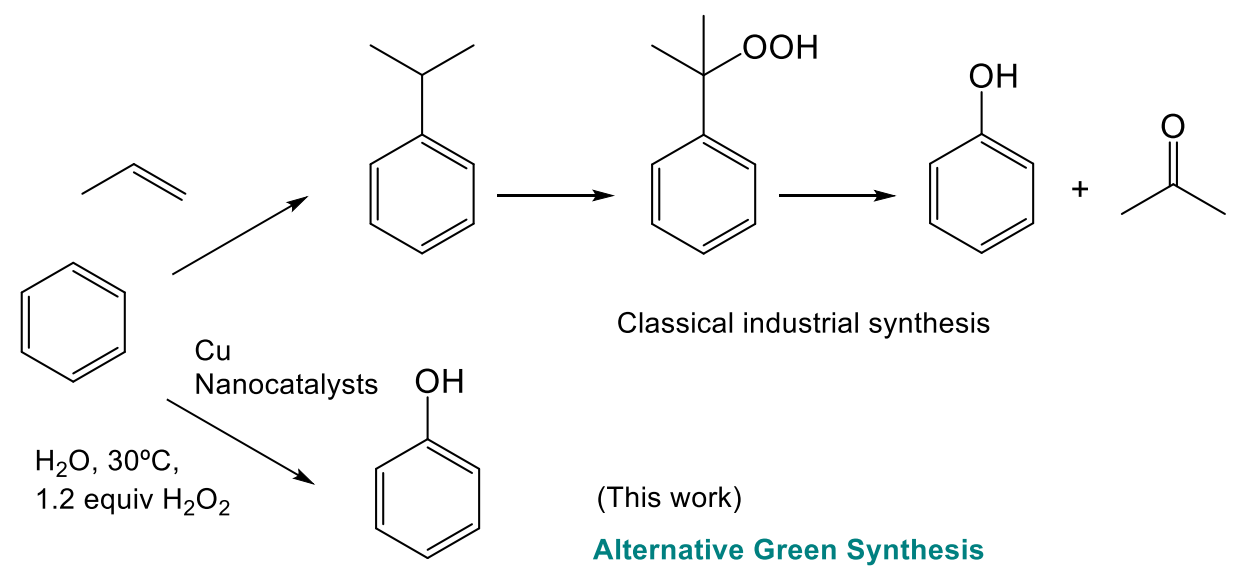

Scheme 1. Synthesis of phenol by selective oxidation of benzene. 


\section{Results and discussion}

\section{Preparation and characterization of different CuNPs-CALB nanobiohybrids.}

The synthesis of nanobiohybrids containing copper nanoparticles have been performed in aqueous media by adding the commercial lipase B from Candida antarctica (CAL-B, $33 \mathrm{kDa}$, monomeric enzyme, supplied by Novozymes) to an aqueous solution of fully water soluble copper salt at room temperature and under gentle stirring (Scheme 2). The copper (II) sulfate pentahydrate $\left[\mathrm{Cu}_{2} \mathrm{SO}_{4} \times 5 \mathrm{H}_{2} \mathrm{O}\right]$ was used as copper salt with a concentration of $10 \mathrm{mg} / \mathrm{mL}$. $1.8 \mathrm{~mL}$ of CAL-B was added to $60 \mathrm{~mL}$ of copper salt solution at room temperature. Different buffers and different concentrations were tested and the use of $100 \mathrm{mM}$ of sodium bicarbonate $(\mathrm{pH} \mathrm{10)}$ and $100 \mathrm{mM}$ of sodium phosphate $(\mathrm{pH} 7)$ were the best options.

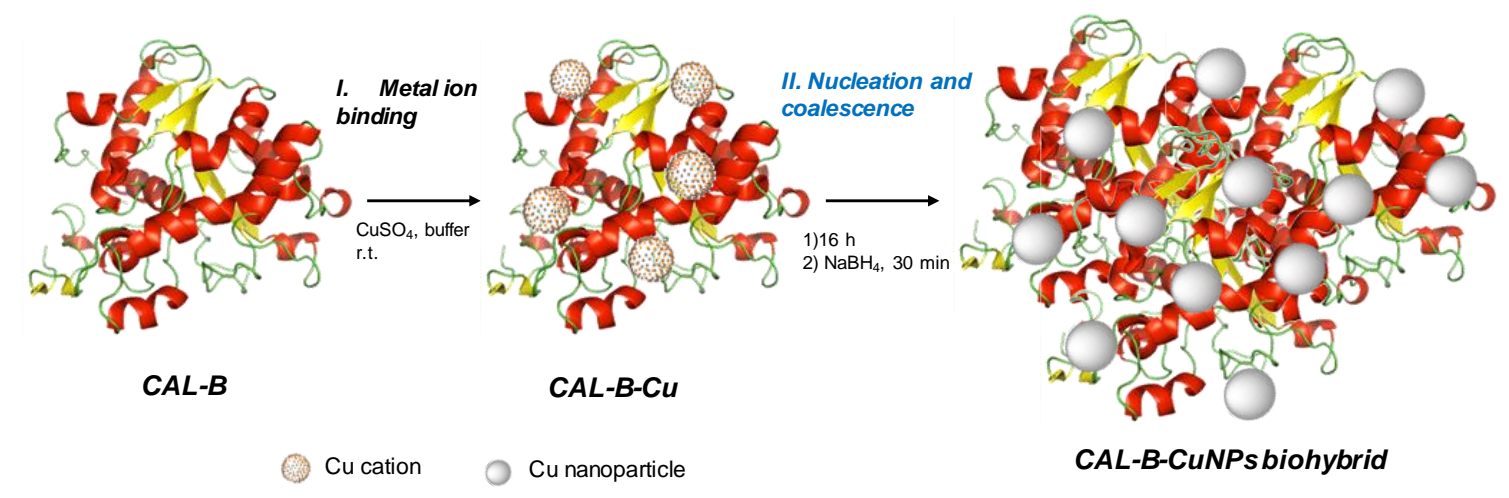

Scheme 2. Experimental workflow to prepare the novel heterogeneous CAL-B-CuNPs nanobiohybrids. I) Rapid copper binding to the protein in presence of buffer solution and $\mathrm{Cu}(\mathrm{ii})$, II) Precipitation of the composite, rapid metal reduction and nanoparticle growing.

After $16 \mathrm{~h}$ incubation, the solid was reduced by using sodium borohydride, washed several times with distilled water, centrifuged and lyophilized overnight to obtaining the two heterogeneous biohybrids Cu-CALB-BIC and Cu-CALB-PHOS respectively 
(Scheme 2). Both catalysts were characterized by different analysis techniques such as XRD, ICP-OES, TEM and SEM. Different species were obtained depending on the buffer used in the synthesis.

XRD analysis demonstrated that the main copper species in Cu-CALB-BIC hybrid was $\mathrm{Cu}(0)$, containing around $20 \%$ of $\mathrm{Cu}(\mathrm{i})$ in form of $\mathrm{Cu}_{2} \mathrm{O}$ (Figure 1). TEM analysis

A

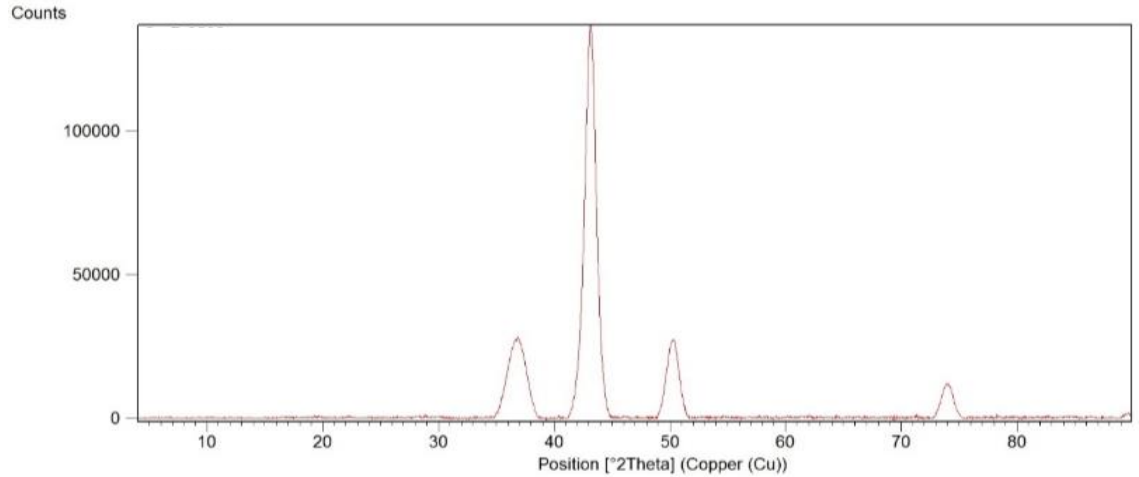

B

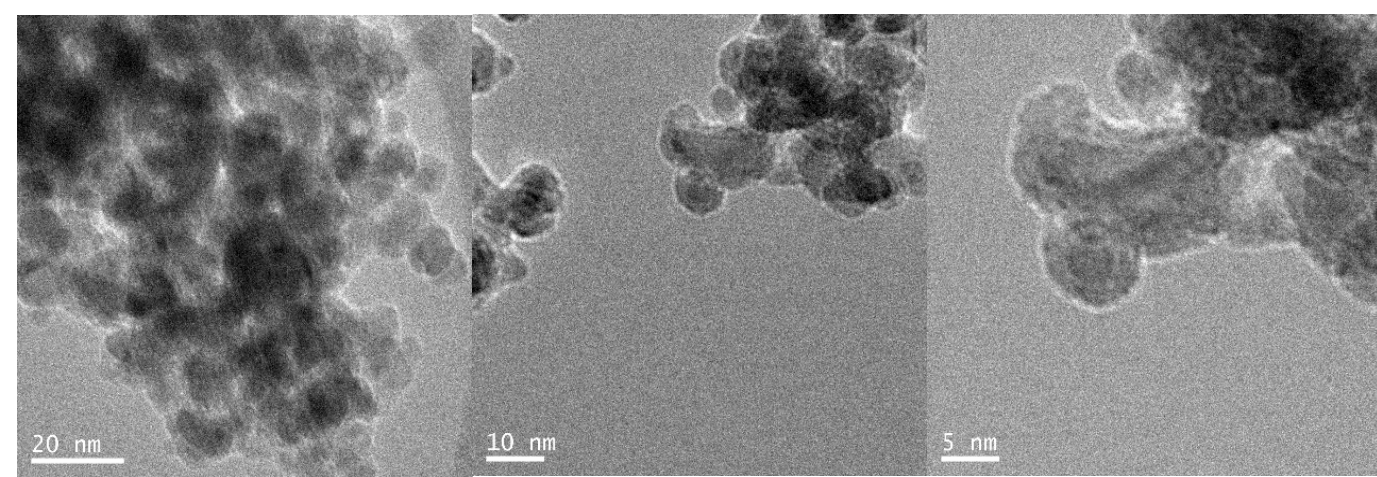

Figure 1. Characterization of the Cu-CALB-BIC biohybrid. A) XRD spectrum, B) TEM images.

showed the formation of nanoparticles of around $9 \mathrm{~nm}$ diameter size (Figure 1B). ICPOES showed than the content of $\mathrm{Cu}$ in the Cu-CALB-BIC biohybrid was $84 \%$. Using heating at $100^{\circ} \mathrm{C}$ for drying instead of lyophilization, the $\mathrm{Cu}_{2} \mathrm{O}$ increased to be around $60 \%$ of $\mathrm{Cu}$ species, the rest was $\mathrm{Cu}(0)$ (Figure $\mathrm{S} 1$ ).

In the case of Cu-CALB-PHOS hybrid, XRD showed than the main $\mathrm{Cu}$ species was $\mathrm{Cu}(\mathrm{i})$ in form of $\mathrm{Cu}_{2} \mathrm{O}$ around $70 \%$ and also containing around $30 \%$ of $\mathrm{Cu}(0)$ (Figure 
2). This biohybrid presented $81 \% \mathrm{Cu}$ content calculated by ICP-OES analysis. TEM experiments revealed the formation of dispersed nanoparticles of $15 \mathrm{~nm}$ (Figure 2B), slightly larger than with using bicarbonate as buffer of synthesis (Figure 1). Also this method was modified by using acetone in the washing step instead of water, or heating at $80^{\circ} \mathrm{C}$ instead of lyophilization for obtaining the solid. In all these conditions the nanobiohybrids presented mixture of the different cu species and the catalytic properties and mechanical stability of the solid were worse (data not shown).

A test applying the synthetic protocol directly in distilled water without using buffer in the solution was also performed. At these conditions, XRD pattern showed that mixture of $\mathrm{Cu}(\mathrm{i})$ as $\mathrm{Cu}_{2} \mathrm{O}$ and $\mathrm{Cu}(0)$ was obtained (Figure S2), and ICP-OES determined an amount of $68 \%$ cooper in the sample.

A

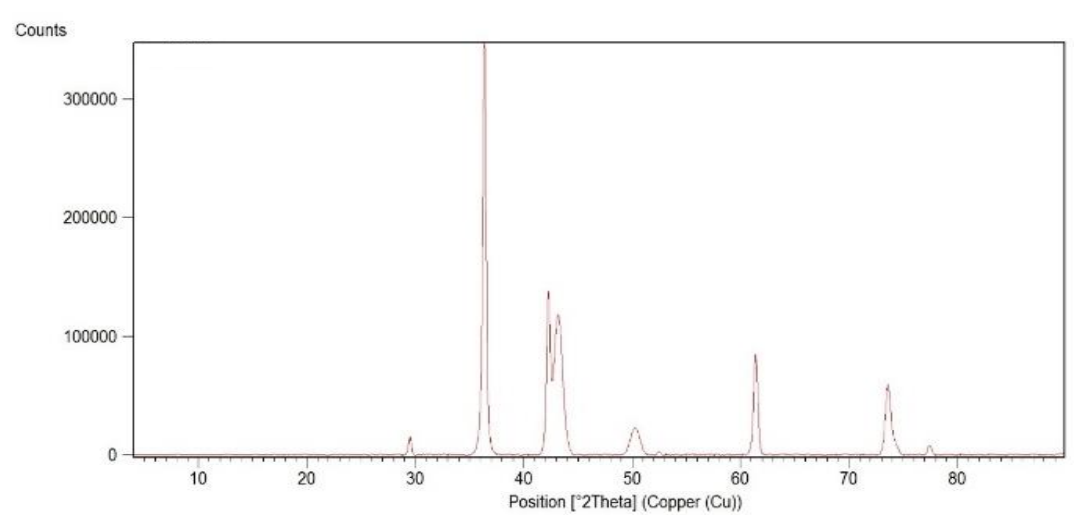

B
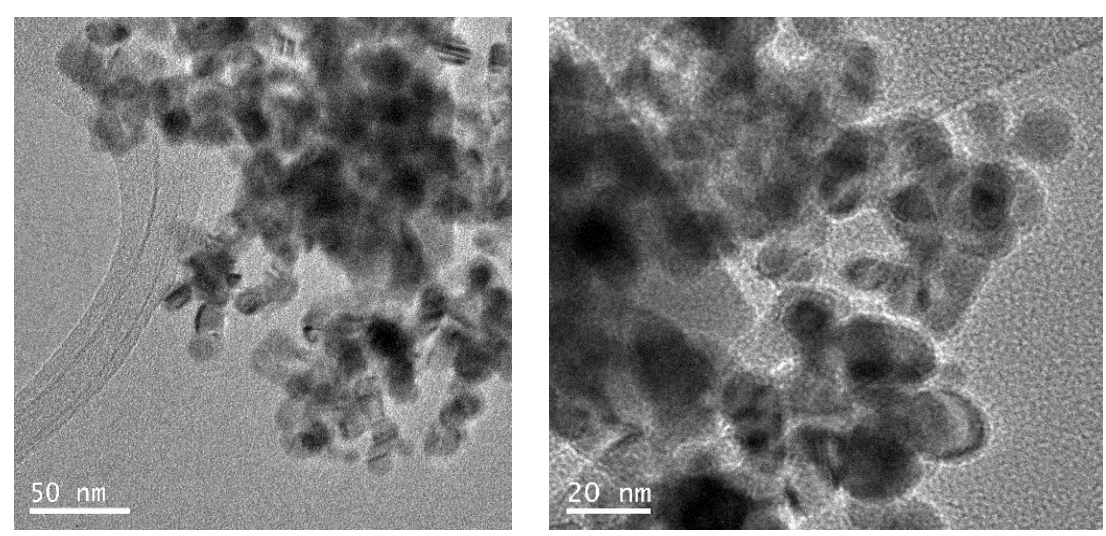

Figure 2. Characterization of the Cu-CALB-PHOS biohybrid. A) XRD spectrum, B) TEM images. 
One modification on the previous protocols was adding the double amount of enzyme in the preparation maintaining the rest steps the same. This modification did not affect to the copper species in method using carbonate as buffer, where XRD pattern of the socalled nanohybrid $\mathbf{C u}-\mathrm{CALB}-\mathrm{BIC2}$ showed the characteristic peaks of $\mathrm{Cu}(0)$ and one minority at $37^{\circ}$ of the $\mathrm{Cu}_{2} \mathrm{O}$ (thus was slightly lower than in $\mathbf{C u}-\mathbf{C A L B}-\mathbf{B I C}$ ) (Figure 3). However, the content of $\mathrm{Cu}$ in this sample was $93 \%$, around $10 \%$ more than using half amount of protein (Cu-CALB-BIC). TEM analysis revealed the formation of $\mathrm{Cu}$ (0) NPs as a core of $6 \mathrm{~nm}$ (Figure 3), smaller size than observed in Cu-CALB-BIC (Figure 1). In the case of using phosphate as buffer, differences in cu species and nanoparticles size were observed. In this case, XRD pattern determined that Cu-CALBPHOS-2 biohybrid showed peaks exclusively corresponding to $\mathrm{Cu}_{2} \mathrm{O}$ species, without traces of $\mathrm{Cu}(0)$ (Figure 4). Also changes in the amount of $\mathrm{Cu}$ was observed, being for this hybrid

A

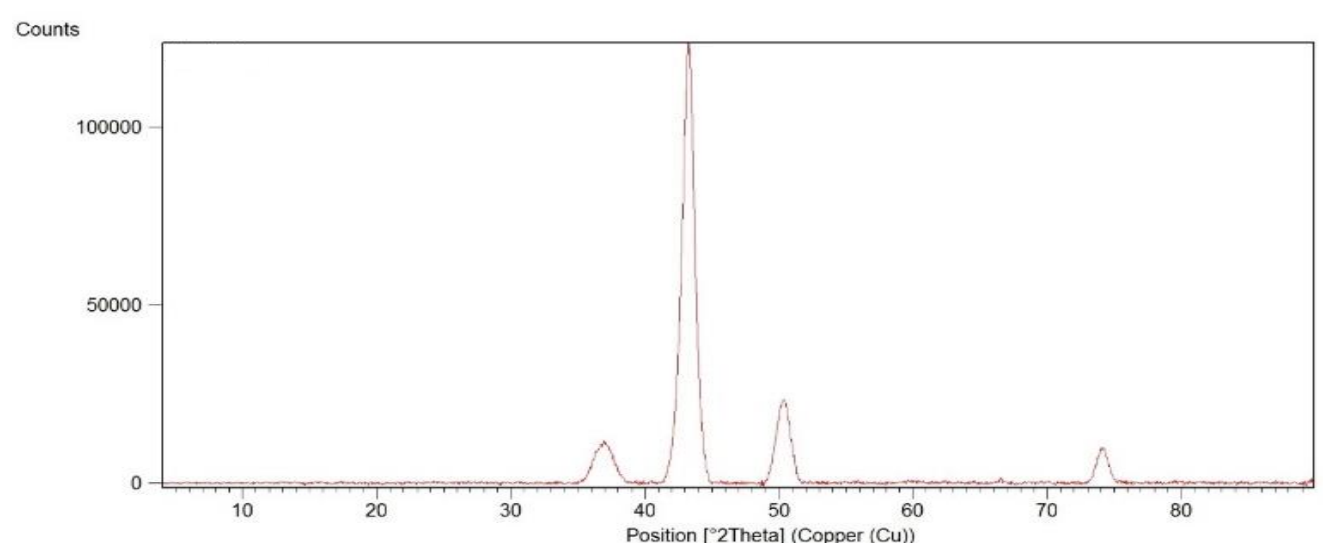

B

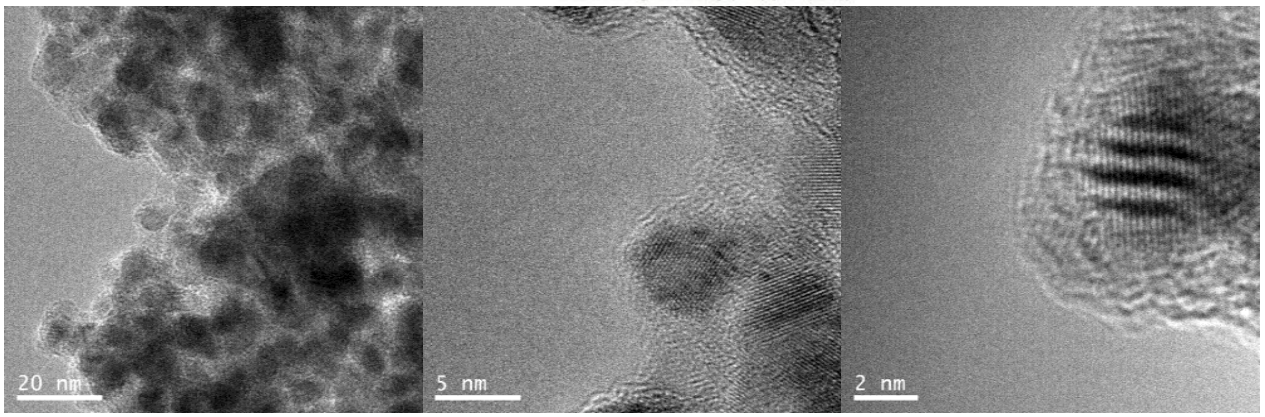

Figure 3. Characterization of the Cu-CALB-BIC-2 biohybrid. A) XRD spectrum, B) TEM images. 
$61 \%$ determined by ICP-OES, 20\% less than in Cu-CALB-PHOS. TEM analysis also exhibited the formation of crystalline nanoparticles with a diameter size of $10 \mathrm{~nm}$ (Figure 4).

Therefore, by using this last methodology, a novel $\mathrm{Cu}$ nanobiohybrid of controlled morphology, size and metal species was synthesized. This could be explained by the concept of larger amount of protein influence controlling the reduction of the cooper oxide species and also influenced in the coalescence step, controlling the nanoparticle growth.

Furthermore, selecting the methodology using phosphate buffer, other types of $\mathrm{Cu}$ nanobiohybrids were synthesized avoiding the reducing step (Cu-CALB-NR) or changing it by incubation in the presence of hydrogen peroxide (oxidative step) (Cu-

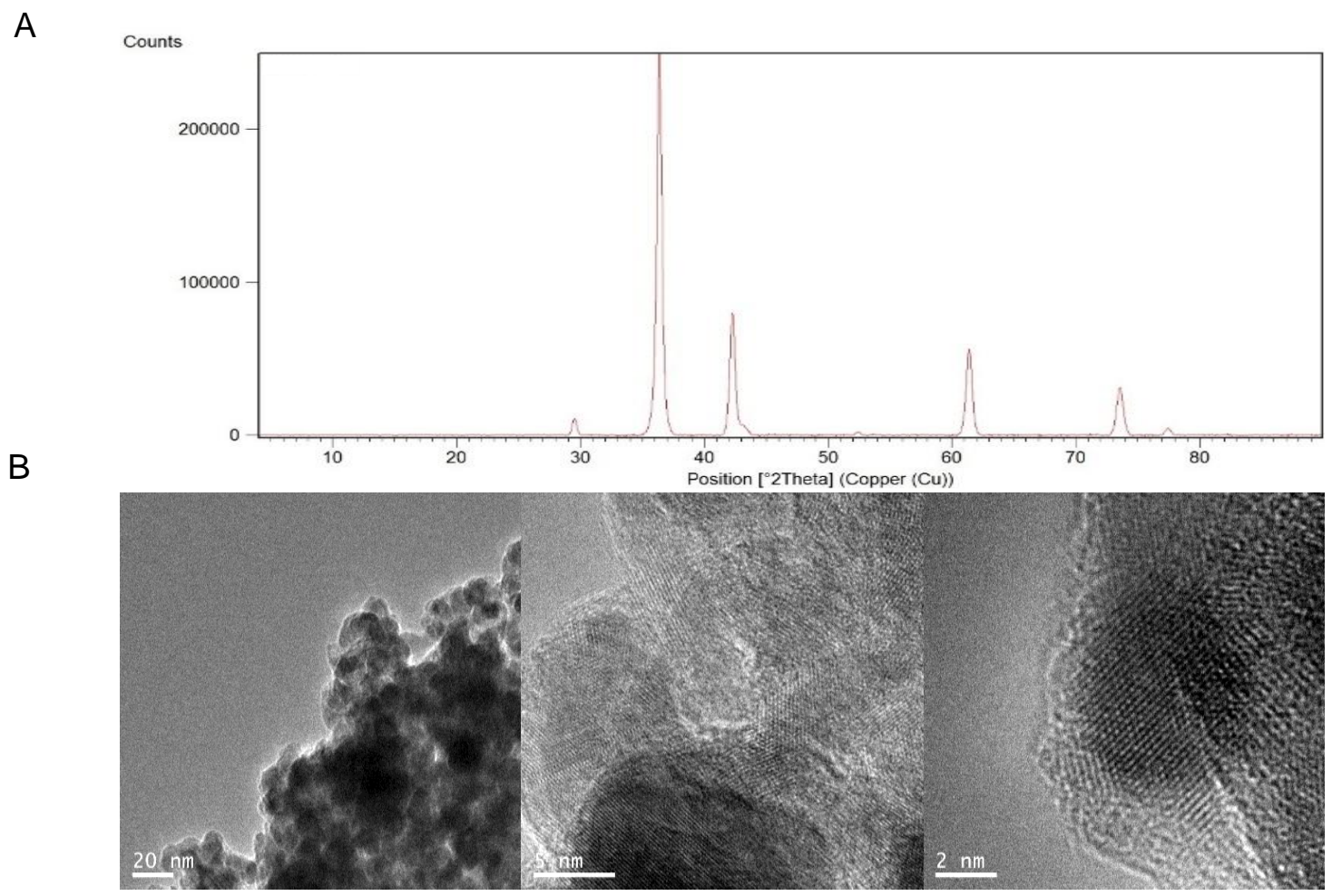

Figure 4. Characterization of the Cu-CALB-PHOS-2 biohybrid. A) XRD spectrum, B) TEM images. 
CALB-NRH $\left.\mathbf{O}_{2} \mathrm{O}_{2}\right)$ or $\mathrm{NaOH}(\mathrm{Cu}-\mathrm{CALB}-\mathbf{N R N a O H})$ in the methodology. In the three cases, a light blue solid was obtained instead of the typical black colour for the other biohybrids. $\mathrm{XRD}$ showed that in this case the copper species were $\mathrm{Cu}_{3}\left(\mathrm{PO}_{4}\right)_{2}$ in all cases (Figures 5, S3-S4) and the $\mathrm{Cu}$ content were $32 \%, 22 \%$ and $35 \%$, respectively. TEM analysis revealed the slightly differences on the diameter size of the nanoparticles. In all cases crystalline spherical nanoparticles were formed, from 3-5 nm in Cu-CALB-NR (Figure 5), $5 \mathrm{~nm}$ for CALB-NRH $\mathbf{O}_{2}$ (Figure S3), and $5.8 \mathrm{~nm}$ in Cu-CALB-NRNaOH (Figure S4).

A
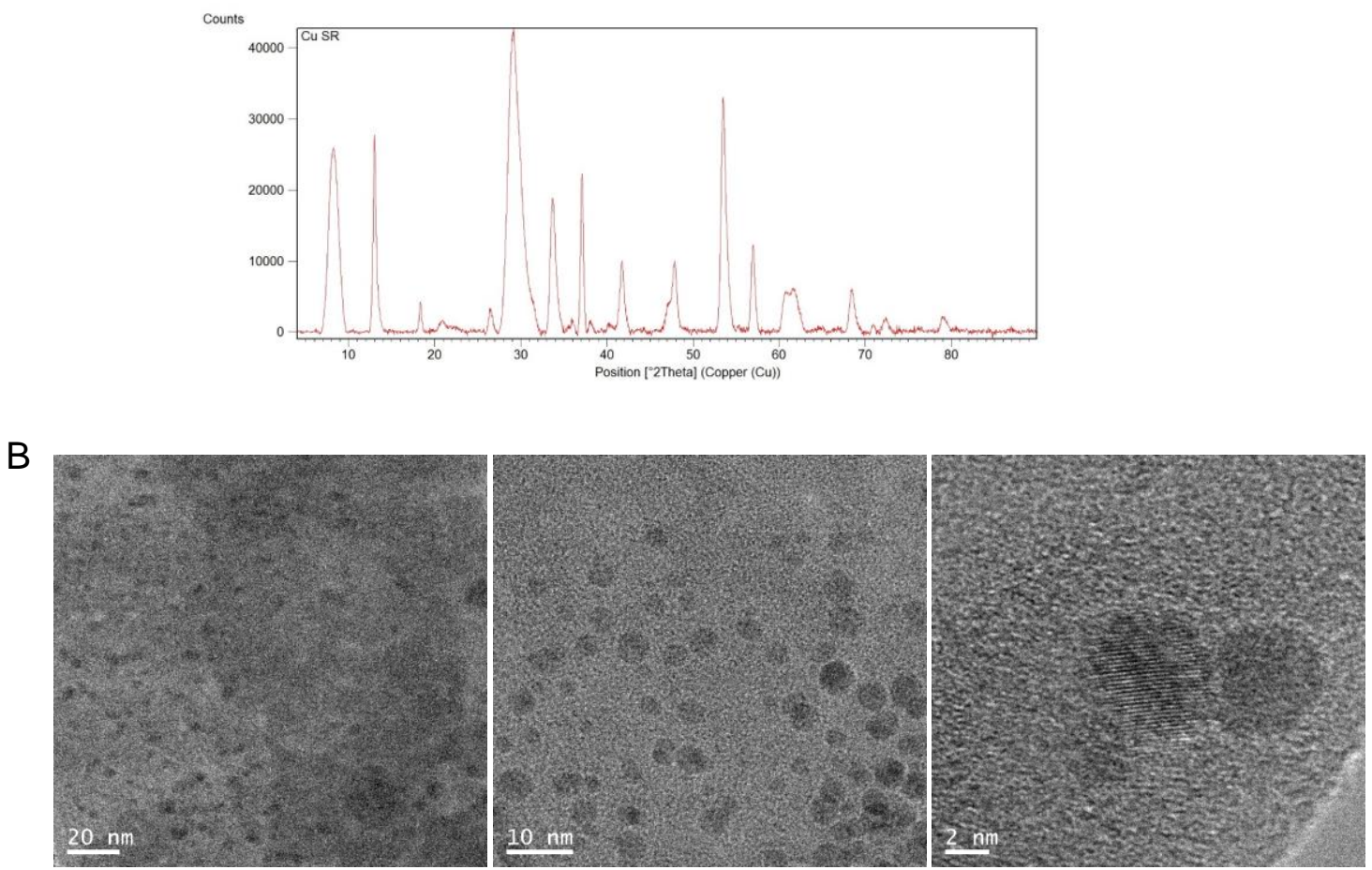

Figure 5. Characterization of the Cu-CALB-PHOS-NR biohybrid. A) XRD spectrum, B) TEM images.

Finally, a decrease in the amount of sodium borohydride used in the reduction step was performed. $10 \%(\mathrm{w} / \mathrm{v})$ of $\mathrm{NaBH}_{4}$ used in the previous method was added in this case maintaining intact the rest of synthetic steps in the method of synthesis of Cu-CALBPHOS, in this case the biohybrid called Cu-CALB-PHOS10\% R. At these synthetic 
conditions, a biohybrid constituted of extremely crystalline $\mathrm{Cu}_{3}\left(\mathrm{PO}_{4}\right)_{2}$ nanoparticles (diameter size approx. $10 \mathrm{~nm}$ ) was obtained (Figure 6) instead of the $\mathrm{Cu} / \mathrm{Cu}_{2} \mathrm{O} \mathrm{NPs}$ biohybrid synthesized in Cu-CALB-PHOS (Figure 2). Also the $\mathrm{Cu}$ content in this case was $48 \%$ instead of $81 \%$ with the full reduction.

A final test using ten times more amount of protein and increasing the incubation time from $24 \mathrm{~h}$ to $72 \mathrm{~h}$ was performed. These variations were used in two protocols, using phosphate buffer and with or without reduction step, synthesizing the Cu-CALBPHOS-10 and Cu-CALB-PHOS-NR-10 bionanohybrids respectively.

A

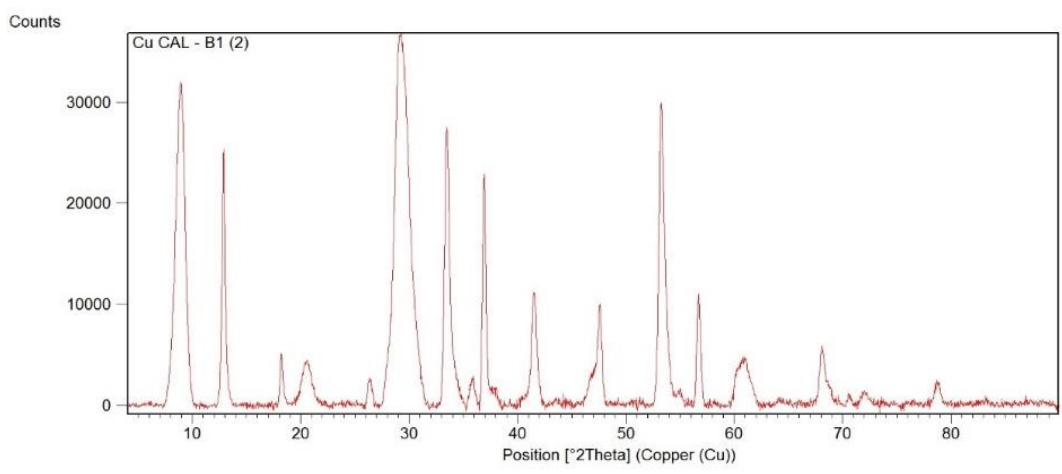

B

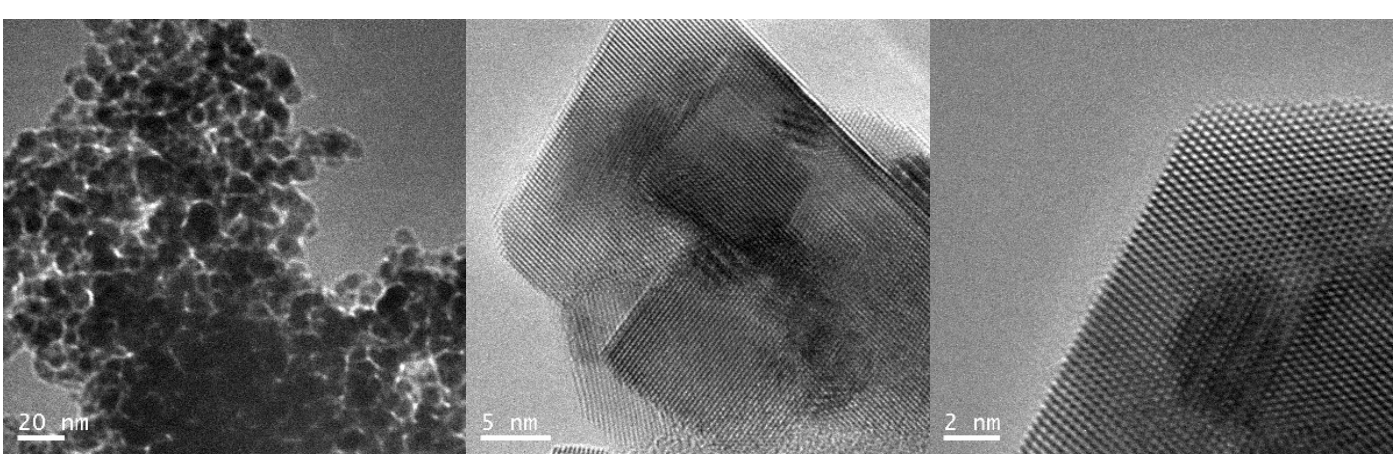

Figure 6. Characterization of the Cu-CALB-PHOS10\% biohybrid. A) XRD spectrum, B) TEM images.

In this case SEM analysis revealed the formation of well-formed nanoflowers in both cases (Figure S5-S6). XRD pattern showed the presence of $\mathrm{Cu}_{3}\left(\mathrm{PO}_{4}\right)_{2}$ as main copper species, containing also $\mathrm{Cu}_{2} \mathrm{O}$ specially in Cu-CALB-PHOS-10 (Figure S5) where 
reduction step was used. ICP-OES determined a content of $\mathrm{Cu}$ of around $50 \%$ in each case.

In all cases, the cu nanobiohybrids were synthesized by a very effective, simple and sustainable way at multimilligram scale, easily scalable to grams.

\section{Determination of catalytic activity pNP reduction}

In order to test the metallic activity of the novel cu nanobiohybrids, the activity in the reduction of p-nitrophenol (pNP) to p-aminophenol (pAP) in aqueous media and room temperature was performed as model reaction (Table 1).

Table 1. Reduction of pNP to pAP by all bionanohybrids.

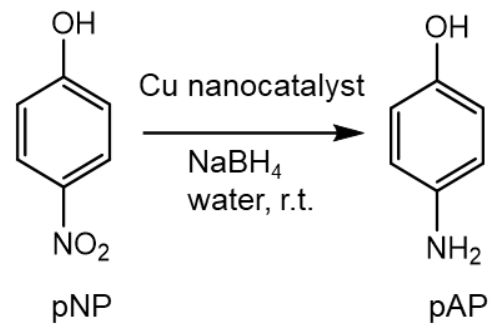

\begin{tabular}{ccc}
\hline Cu-Bionanohybrid & Time $(\mathrm{min})^{\mathrm{a}}$ & $\mathrm{TOF}^{\left(\mathrm{min}^{-1}\right)^{\mathrm{b}}}$ \\
\hline Cu-CALB-PHOS & 1.5 & 4.85 \\
Cu-CALB-BIC & 0.5 & 14.04 \\
Cu-CALB-PHOS-2 & 3 & 3.28 \\
Cu-CALB-BIC-2 & 0.5 & 12.55 \\
Cu-CALB-PHOS-NR & 4 & 4.59 \\
Cu-CALB-PHOS-NR-NaOH & 3 & 5.62 \\
Cu-CALB-PHOS-NR-H2O2 & 3 & 8.95 \\
Cu-CALB-PHOS10\% R & 1.5 & 8.18 \\
Cu-CALB-PHOS-10 & 12.5 & 0.94 \\
Cu-CALB-PHOS-NR-10 & 15 & 0.78
\end{tabular}

${ }^{a}$ Time at all pNP was transformed in pAP. ${ }^{\mathrm{b}}$ The TOF value was defined as the moles of pAP per mole of copper atoms in the nanobiohybrid per minute. 
Cu-CALB-BIC and Cu-CALB-BIC-2 (mainly $\mathrm{Cu}(0)$ ) were the faster nanocatalysts with a complete transformation of pNP to pAP (150 ppm) in 30 seconds (Figure S7), with a highest TOF value of $14.04 \mathrm{~min}^{-1}$ for Cu-CALB-BIC (Table 1). However, Cu nanohybrids containing $\mathrm{Cu}(\mathrm{I})$ species showed lower catalytic efficiency, Cu-CALBPHOS showed a TOF value of $8.85 \mathrm{~min}^{-1}$, slightly higher than the results obtained using Cu-CALB-PHOS-2. This result could be explained considering that this latter catalyst present exclusively $\mathrm{Cu}(\mathrm{I})$ species whereas the former also contain $\mathrm{Cu}(0)$ (Table 1$)$. The non-reduced biohybrid Cu-CALB-PHOS-NR showed similar TOF value in the reduction than Cu-CALB-PHOS whereas the two modifications Cu-CALB-PHOSNR-NaOH, Cu-CALB-PHOS-NR- $\mathrm{H}_{2} \mathrm{O}_{2}$ showed better performance than the former, even almost two fold in the latter with a TOF value of $8.95 \mathrm{~min}^{-1}$, the best results after the biohybrids containing high amount of $\mathrm{Cu}(0)$. Also this last value could corroborate the results obtained using Cu-CALB-PHOS10\%R (lower reduction) which showed almost double TOF value than the Cu-CALB-PHOS. Finally, the $\mathrm{Cu}$ biohybrids containing nanoflowers (Cu-CALB-PHOS-10, Cu-CALB-PHOS-NR-10) showed very low catalytic efficiency in this reaction with TOF values $<1 \mathrm{~min}^{-1}, 15$ times less than the Cu-CALB-BIC biohybrid.

\section{Selective oxidation of benzene to phenol catalyzed by different CuNPs- CALB biohybrids}

The different synthesized CuNPs biohybrids were evaluated as catalysts in a $\mathrm{C}-\mathrm{H}$ activation process, the direct selective oxidation of benzene to phenol at mild conditions. The reaction was performed in aqueous media using a green oxidant (hydrogen peroxide) at $25^{\circ} \mathrm{C}$. Initially, for the study, the biohybrid Cu-CALB-PHOS-2 was selected. The effect of the addition different equivalents of hydrogen peroxide was tested (Figure S8). Very low conversion (1\%) was obtained using 1.25-1.5 equiv of 
$\mathrm{H}_{2} \mathrm{O}_{2}$ with $95 \%$ selectivity in phenol formation. However, the addition of 5 equiv of oxidant resulted in an increase of conversion up to $22 \%$, although decreasing the selectivity in the phenol formation, hydroquinone and benzoquinone as subproducts were observer. The increase up to 10 equiv caused a negative effect, with a decrease in conversion $6 \%$ (4.8\% phenol) but more important decreasing the selectivity value to 73\% (Figure S8). These results corroborate also at these conditions previous some works ${ }^{34}$ where it has been reported the negative effect on the selectivity of the reaction by addition of high amount of this oxidant. Therefore, 1.25 equiv was selected as amount to use.

Thus, considering the low conversion obtained in pure water, we evaluate the effect of adding acetonitrile as co-solvent in the reaction medium at room temperature using 1.25 equiv of $\mathrm{H}_{2} \mathrm{O}_{2}$ (Table $\mathrm{S} 2$ ). Thus, when a very short amount of acetonitrile $(1 \% \mathrm{v} / \mathrm{v})$ was added the yield increased 3 times with an extremely high selectivity (>99\%) (Table S2). Higher amount of acetonitrile was added from 20 to $40 \%$ caused a decrease in yield but more important in the selectivity on the unique production of phenol, especially at $40 \%$ of acetonitrile where the reaction was 2 times slower than in pure water with a very low selectivity (26\%) (Table S2). Considering the conditions selected as low amount of oxidant and co-solvent in the mixture, we tested to increase the temperature from 25 to $30^{\circ} \mathrm{C}$. Surprisingly this slight increase in five degrees caused an important increase in the yield of phenol, from $3 \%$ to almost $28 \%$ maintaining the excellent selectivity (99\%) (Figure S9). This constitutes a very promising results because it was possible to producing almost $30 \%$ of pure phenol from benzene at very environmentally sustainable conditions.

Therefore, once the conditions were optimized the rest of CuNPs biohybrids were tested in order to evaluate their catalytic performance in this $\mathrm{C}-\mathrm{H}$ activation process (Table 2). 
Table 2. Selective oxidation of benzene catalyzed by different CuNPs-CALB biohybrids. $^{[\mathrm{a}]}$

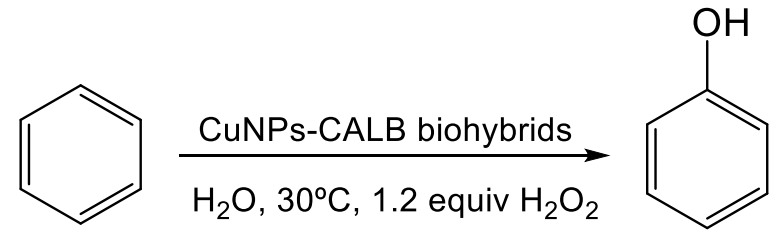

\begin{tabular}{cc}
\hline $\mathrm{Cu}$ & $\mathrm{C}^{\mathrm{c}}$ \\
Bionanohybrid & $(\%)$ \\
\hline- & 0 \\
$\mathrm{Cu}^{-\mathrm{PHOS}^{\mathrm{b}}}$ & 0 \\
$\mathrm{Cu}-\mathrm{BIC}^{\mathrm{b}}$ & 0
\end{tabular}

$\begin{array}{ccccc}\text { Cu-CALB-PHOS } & 7 & 7 & 99 & 45 \\ \text { Cu-CALB-BIC } & 2 & 2 & 99 & 17.5 \\ \begin{array}{c}\text { Cu-CALB-PHOS-2 } \\ \text { Cu-CALB-BIC-2 }\end{array} & 28 & 28 & 99 & 244 \\ \text { Cu-PHOSNR } & 2.75 & 2.75 & 99 & 15 \\ \text { Cu-CALB-PHOS- } & 9 & 9 & 90 & \text { nd } \\ \quad \text { NR } & 69 & 66 & 95 & 1,050 \\ \begin{array}{c}\text { Cu-CALB-PHOS- } \\ \text { NR-NaOH }\end{array} & 87 & 81 & 95 & 1,200 \\ \begin{array}{c}\text { Cu-CALB-PHOS- } \\ \text { NR-H2O2 }\end{array} & 68 & 59 & 87 & 1,400 \\ \begin{array}{c}\text { Cu-CALB- } \\ \text { PHOS10\%R }\end{array} & 75 & 72 & 96 & 780 \\ \end{array}$

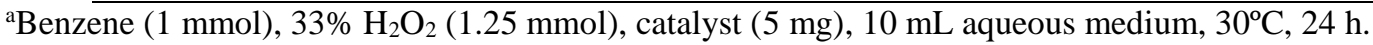

${ }^{\mathrm{b}}$ Sample of biohybrids prepared without enzyme using the protocol described. ${ }^{\mathrm{c}} \mathrm{C}$ : conversion of benzene. ${ }^{\mathrm{d}}$ Phenol yield was calculated by HPLC. ${ }^{\mathrm{e}}$ Selectivity of phenol respect the formation of other subproducts (benzoquinone and hydroquinone) was determined by HPLC. ${ }^{\mathrm{f}}$ TOF value $\left(\mathrm{h}^{-1} \times 10^{-3}\right)$ was defined as the moles of phenol produced per mole of copper atoms in the nanobiohybrid per minute.

The results in Table 2 showed a clear influence of the type of CuNPs (cu species, nanoparticle size) in the final rate and selectivity in the hydroxylation process. The catalytic performance of all $\mathrm{Cu}$ nanocatalysts were compared at $24 \mathrm{~h}$ reaction. 
Very low conversions were achieved for the biohybrids containing $\mathrm{Cu}(0)$, being the best one Cu-CALB-PHOS with 7\% conversion and excellent selectivity (>99\%). However, Cu-CALB-PHOS-2 $\left(\mathrm{Cu}_{2} \mathrm{O}\right.$ NPs) showed a relative good conversion compared with many reported catalysts- $28 \%$ conversion of benzene with a selectivity of $>99 \%$ in phenol production in aqueous media and $30^{\circ} \mathrm{C}$ (Table 2). Nevertheless, the non or low-reduced catalysts, containing $\mathrm{Cu}_{3}\left(\mathrm{PO}_{4}\right)_{2}$ nanoparticles, showed the highest values in catalytic conversion between 3 to 6 times higher than using Cu-CALBPHOS-2 (Table 2). The highest conversion of benzene was obtained using Cu-CALBPHOS-NR-NaOH, with $87 \%$ (81\% phenol yield) at $24 \mathrm{~h}$, with a selectivity of $94 \%$ (Table 2). This catalyst produced phenol at $11 \%$ in $6 \mathrm{~h}$ with selectivity $>99 \%$ (Table S3). Using Cu-CALB-PHOS-NR biohybrid, 69\% conversion of benzene was achieved with a selectivity of $95 \%$ on phenol production, with was of $98 \%$ selectivity at $17 \mathrm{~h}$ (Table S3). The conversion was similar to the latter using Cu-CALB-PHOS-NR-H2 $\mathbf{H}_{2}$ although a slight decrease in selectivity was observed (Table 2). The catalyst with low reduction step, Cu-CALB-PHOS10\% $\mathbf{R}$ also showed a very good results, catalysing the conversion of benzene at $75 \%$ with a phenol selectivity of $96 \%$ (table 2).

The reaction was also tested using Cu-CALB-PHOS-NR-NaOH at $45^{\circ} \mathrm{C}$ (Figure S10). Although the conversion of benzene was increased up to $97 \%$, the selectivity of phenol production slightly decreased.

Therefore, these are the best results in term of conversion and selectivity of benzene to phenol in water and low temperature as far as we known. 


\section{Conclusions}

Novel $\mathrm{Cu}$ nanoparticles-enzyme biohybrids, in situ Controlled-Cu species and nanoparticle size in a protein host network, have been synthesized at multimilligram scale from the direct combination of an enzyme and a copper salt in aqueous media at room temperature. The $\mathrm{Cu}$ species, size, crystallinity and morphology of the nanoparticles has been easily controlled by particular modifications on the protocol described, for example buffer used on the solution, $\mathrm{pH}$ or time in the reducing step, being possible to obtaining bionanohybrids containing very small size $\mathrm{Cu}(0) \mathrm{NPs}$, exclusively $\mathrm{Cu}_{2} \mathrm{O}$ NPs or very crystalline $\mathrm{Cu}_{3}\left(\mathrm{PO}_{4}\right)_{2}$ NPs. Some of these synthesized CuNPs biohybrids, specially containing $\mathrm{Cu}(0)$, showed good activity again reduction process.

Very excellent results were achieved in the selective oxidation of benzene in water at $30^{\circ} \mathrm{C}$ using hydrogen peroxide as green oxidant. This $\mathrm{C}-\mathrm{H}$ activation process was performed, depending on the cu-biohybrid used as nanocatalysts, at $>80 \%$ benzene conversion with very high phenol selectivity (>95\%), being catalysts containing $\mathrm{Cu}_{3}\left(\mathrm{PO}_{4}\right)_{2} \mathrm{NPs}$ in principle as the best ones.

As far as we know, this is the best result in yield and selectivity of direct transformation of phenol from benzene using aerobic oxidation and green oxidant in water.

The very simple and green technology for synthesizing these excellent heterogeneous catalysts in high amount represent a challenge in this very important reaction with a further application in the extension of their catalytic performance in other $\mathrm{C}-\mathrm{H}$ activation reactions. 


\section{Experimental Section}

\section{Experimental Section}

\section{General}

Lipase B from Candida antarctica (CAL-B) solution was purchased from Novozymes (Copenhagen, Denmark). Copper (II) sulfate pentahydrate $\left[\mathrm{Cu}_{2} \mathrm{SO}_{4} \times \mathrm{H}_{2} \mathrm{O}\right]$ and hydrogen peroxide (33\%) were from Panreac (Barcelona, Spain). P-nitrophenol, pnitrophenyl propionate, sodium bicarbonate, sodium phosphate, sodium borohydride, phenol, benzoquinone, hydroquinone and catechol were purchased from Sigma-Aldrich (St. Louis, MO, USA). HPLC grade acetonitrile was from Scharlab (Barcelona, Spain). Inductively coupled plasma - optical emission spectrometry) (ICP-OES) was performed on a OPTIMA 2100 DV instrument (PerkinElmer, Waltham, MA, USA). X-Ray diffraction (XRD) patterns were obtained using a Texture Analysis D8 Advance Diffractometer (Bruker, Billerica, MA, USA) with $\mathrm{Cu} \mathrm{K \alpha}$ radiation. Transmission electron microscopy (TEM) and high resolution TEM microscopy (HRTEM) images were obtained on a 2100F microscope (JEOL, Tokyo, Japan) equipped with an EDX detector INCA x-sight (Oxford Instruments, Abingdon, UK). Interplanar spacing in the nanostructures was calculated by using the inversed Fourier transform with the GATAN digital micrograph program (Corporate Headquarters, Pleasanton, CA, USA). Scanning electron microscopy (SEM) imaging was performed on a TM-1000 microscope (Hitachi, Tokyo, Japan). To recover the biohybrids, a Biocen 22 R (Orto-Alresa, Ajalvir, Spain) refrigerated centrifuge was used. Spectrophotometric analyses were run on a V-730 spectrophotometer (JASCO, Tokyo, Japan). A HPLC pump PU-4180 (JASCO, Tokyo, Japan) was used. Analyses were run at $25{ }^{\circ} \mathrm{C}$ using a RI-4030 refractive Index detector (JASCO, Tokyo, Japan). 


\section{General synthesis of Cu-CALB-BIC and Cu-CALB-PHOS bionanohybrids.}

$1.8 \mathrm{~mL}$ of commercial (18 $\mathrm{mg}$ of protein) Candida antarctica lipase solution was added to $60 \mathrm{~mL}$ buffer $0.1 \mathrm{M}$ (sodium bicarbonate $\mathrm{pH}=10$ or sodium phosphate $\mathrm{pH} 7$ ) in a 250 $\mathrm{mL}$ glass bottle containing a small magnetic bar stirrer. Then, $600 \mathrm{mg}$ of $\mathrm{Cu}_{2} \mathrm{SO}_{4} \mathrm{x}$ $5 \mathrm{H}_{2} \mathrm{O}(10 \mathrm{mg} / \mathrm{ml})$ was added to the protein solution and it was maintained for 16 hours. After the first 30 min incubation, the solution turned cloudy (turquoise) and the $\mathrm{pH}$ solution was measured indicating a decrease to 8 or $6 \mathrm{pH}$ value depending on the buffer used. After $16 \mathrm{~h}, 6 \mathrm{~mL}$ of $\mathrm{NaBH}_{4}(300 \mathrm{mg})$ aqueous solution $(1.2 \mathrm{M})$ was added to the cloudy solution (in two times of $3 \mathrm{~mL}$ ) obtaining a final concentration of $0.12 \mathrm{M}$ of sodium borohydride in the mixture. The solution turned rapidly black and, the mixture was reduced during $30 \mathrm{~min}$. After the incubation, in all cases, the mixture was centrifuged at $8000 \mathrm{rpm}$ for $5 \mathrm{~min}$, (10 mL per falcon type tube). The generated pellet was re-suspended in $15 \mathrm{~mL}$ of water. The $\mathrm{pH}$ of the supernatant solution was measured to be approximately 7 or 9 . It was centrifuged again at 8000 r.p.m for 5 min and the supernatant removed. The $\mathrm{pH}$ of the supernatant solution was measured again, given a $\mathrm{pH}$ value of 7 . The process was repeated twice more. Finally, the supernatant was removed and the pellet of each falcon was re-suspended in $2 \mathrm{~mL}$ of water, collected all solutions in a round-bottom flask, frozen with liquid nitrogen and lyophilized for 16 hours. After that, $150 \mathrm{mg}$ of the so called Cu-CALB-BIC and Cu-CALB-PHOS respectively were obtained.

Different modifications of the protocol were made to obtain different species. Initially, a catalyst was prepared using double amount of enzyme (3.6 mL CALB solution instead of $1.8 \mathrm{~mL}$ ), obtaining Cu-CALB-PHOS-2 and Cu-CALB-BIC-2. Another variation of the protocol was used, in which the reduction step was not performed, obtaining $\mathbf{C u}$ CALB-PHOS-NR. In addition to the last variation, an oxidation step was also 
performed instead of reduction step by the addition of either $6 \mathrm{~mL}$ of a $500 \mathrm{mM}$ solution of sodium hydroxide $(\mathrm{NaOH})$ for $30 \mathrm{~min}$ or $6 \mathrm{~mL}$ of a $0.1 \mathrm{M}$ solution of hydrogen peroxide $\left(\mathrm{H}_{2} \mathrm{O}_{2}\right)$ for 30 min $\left(60 \mu \mathrm{L}\right.$ of the $\mathrm{H}_{2} \mathrm{O}_{2}$ stock solution in $6 \mathrm{~mL}$ of distillate water), obtaining Cu-CALB-PHOS-NRNaOH and Cu-CALB-PHOS-NRH $\mathrm{N}_{2} \mathrm{O}_{2}$ respectively. Another one of the variations was reduction of sodium borohydride at $10 \%$, adding $6 \mathrm{~mL}$ of water containing $\mathrm{NaBH}_{4}(30 \mathrm{mg})$, obtaining $\mathrm{Cu}-\mathrm{CAL}-\mathrm{B}-$

\section{PHOS10\%R.}

The last variation was referred both to the enzyme amount as well as the incubation time, increasing them from $1.8 \mathrm{~mL}$ to $18 \mathrm{~mL}$ in enzyme volume and from $16 \mathrm{~h}$ to $72 \mathrm{~h}$; the obtained catalysts were Cu-CALB-PHOS-10 and Cu-CALB-PHOS-NR-10.

Characterization of the different $\mathrm{Cu}$ bionanohybrids was performed by XRD, ICP-OES (Table S1), TEM and SEM analysis.

\section{Catalytic reduction of 4-nitrophenol (pNP) to 4-aminophenol (pAP)}

To an aqueous solution of p-nitrophenol (pNP) $(1 \mathrm{mM} ; 2 \mathrm{~mL})$, solid $\mathrm{NaBH}_{4}(3 \mathrm{mg})$ was added to reach a final concentration of $0.04 \mathrm{M}$ (The typical catalytic reaction was performed by adding an excess of $\mathrm{NaBH}_{4}$ to ensure its constant concentration throughout the reaction and, therefore, to apply a pseudo-first-order kinetic with respect to the pNP to an aqueous solution of the substrate in the presence of catalysts). In these conditions, upon the addiction of $\mathrm{NaBH}_{4}$, the initial absorbance band of the solution of pNP undergoes to an immediate shift from 317 to $400 \mathrm{~nm}$ due to the formation of 4nitrophenolate ions. Immediately after that, $3 \mathrm{mg}$ of the different Cu-CALB bionanohybrids were added under gentle stirring at $25^{\circ} \mathrm{C}$ in an orbital shaker. The reaction progress was monitored by taking out an aliquot of the solution $(0.1 \mathrm{~mL})$ at 
different times, diluting it with distilled water $(2 \mathrm{~mL})$ and measuring the absorption spectrum between 500 and $300 \mathrm{~nm}$ in a PMMA cuvette (Table 1).

\section{Catalytic oxidation of benzene to phenol.}

Benzene $(88 \mu \mathrm{L}, 1 \mathrm{mmol})$ was added to a $10 \mathrm{~mL}$ water solution containing $1 \%(\mathrm{v} / \mathrm{v})$ acetonitrile. The reaction was vigorously stirring for $10 \mathrm{~min}$. After that, 1.25 equiv of hydrogen peroxide (142 $\mu \mathrm{L}$ of $33 \% \mathrm{v} / \mathrm{v} \mathrm{H}_{2} \mathrm{O}_{2}$ in water) was added to the solution. Finally, $5 \mathrm{mg}$ of nanobiohybrid was added to the mixture to initiate the reaction. Then the mixture was incubated with magnetic stirring at $30^{\circ} \mathrm{C}$ for 24 or $48 \mathrm{~h}$.

The reaction outgoing was monitored by HPLC analysis of the reaction's samples withdrawn at different times. The analysis conditions were performed with a Kromasil$\mathrm{C} 8(150 \times 4.6 \mathrm{~mm}$ and $5 \mu \mathrm{m} \varnothing)$, at a flow of $1.0 \mathrm{~mL} / \mathrm{min}$; and a mobile phase: $50 \%$ (v/v) ACN in MilliQ water, using a refractive index detector (JASCO). Before injection, $30 \mu \mathrm{l}$ of sample was diluted 1:1 in acetonitrile. In these conditions the retention time of benzene was $7.10 \mathrm{~min}$, phenol was 3.20 , benzoquinone 2.70 , hydroquinone was 2.3 and for $\mathrm{H}_{2} \mathrm{O}_{2}$ was $1.4 \mathrm{~min}$. A pure commercial sample of the products were used as standards. The yields were obtained extrapolating the values through a calibration curve of phenol $\left(\mathrm{R}^{2}=0.9901\right)$.

\section{Acknowledgments}

This work was supported by the Spanish Government the Spanish National Research Council (CSIC), SAMSUNG by GRO PROGRAM 2017 and the Ministry of Education, Youth and Sports of the Community of Madrid and the European Social Fund (PEJD- 
2017PRE/SAL-3762). Authors thank the European Cooperation in Science and Technology (COST) program under CA15106 grant (CHAOS: $\mathrm{CH}$ Activation in Organic Synthesis). We also thank Dr. Martinez from Novozymes.

Keywords: Nanoparticles, Heterogeneous biohybrids, Copper, Nanocatalyst, Hydroxylation of benzene.

\section{References}

1) G. Grass, C. Rensing and M. Solioz, Appl. Environ. Microb., 2011,77, 15411547.

2) L. Tamayo, M. Azócar, M. Kogan, A. Riveros and M. Páez, Mat. Sci. Eng. C, 2016,69, 1391-1409.

3) J. Singh, T. Dutta, K.-H. Kim, M. Rawat, P. Samddar and P. Kumar, J. Nanobiotechnol., 2018, 16, art. no. 84.

4) H. Xie, T. Wang, J. Liang, Q. Li and S. Sun, Nano Today, 2018, 21, 41-54.

5) Z. Jia, Y. Li, Z. Zuo, H. Liu, C. Huang, Y. Li, Acc. Chem. Res. 2017, 50, 24702478.

6) H. Chen, J. Motuzas, W. Martens, J.C. Diniz da Costa, App. Catal. B: Environ. 2018, 221, 691-700.

7) K. Zhao, L. Duan, S. Xu, J. Jiang, Y. Fu, Z. Gu, Chem, 2018, 4, 599-612.

8) M.D. Marcinkowski, M.T. Darby, J. Liu, J.M. Wimble, F.R., Lucci, S. Lee, A. Michaelides, M. Flytzani-Stephanopoulos, M. Stamatakis, E.C.H. Sykes, Nat. Chem., 2018, 10, 325-332.

9) X-X Guo, D-W. Gu, Z. Wu, Chem. Rev. 2015, 115, 1622-1651.

10) A. Gawande, M.B. Goswami, F.-X. Felpin, T. Asefa, X. Huang, R. Silva, X. Zou, R. Zboril, R.S. Varma, Chem. Rev. 2016, 116, 3722-3811.

11) S. Shylesh, V. Schnemann, W. R. Thiel, Angew. Chem. Int. Ed. 2010, 49, 342359.

12)F. Zaera, Chem. Soc. Rev. 2013, 42, 2746-2762.

13) G. Li, X. H. Li, Z. J. Zhang, Prog. Chem. 2011, 23, 1644-1656. 
14) J. Mondal, A Biswas, S. Chiba, Y. Zhao, Sci. Rep. 2015, 5, 8294.

15) A. P. LaGrow, L. Sinatra, A. Elshewy, K. W. Huang, K Katsiev, A. R. Kirmani, A. Amassian, D. H. Anjum, O. M. Bakr, J. Phys. Chem. C. 2014, 118, 19374-19379.

16) A. X. Wang, D. Q. Chu, L. M. Wang, B. G. Mao, H. M. Sun, Z.C. Ma, RSC Adv. 2014, 4, 7545-7548.

17) S. R. Ye, A. R. Rathmell, I. E. Stewart, Y. C. Ha, A. R. Wilson, Z. F. Chen, B. J. Wiley, Chem. Commun. 2014, 50, 2562-2564.

18)F. Cui, Y. Yu, L. Dou, J. Sun, Q. Yang, C. Schildknecht, K. Schierle-Arndt, P. Yang, Nano Lett. 2015, 15, 7610-7615.

19) J. C. Yu, F. G. Zhao, W. Shao, C. W. Ge, W. S. Li, Nanoscale 2015, 7, 8811-8818.

20) M. A. Bhosale, T. Sasaki, B. M. Bhanage, Catal. Sci. Technol. 2014, 4, 4274-4280.

21) G. G. Jang, C. B. Jacobs, R. G. Gresback, I. N. Ivanov, H. M., Meyer, M. Kidder, P. C. Joshi, G. E. Jellison, T. J. Phelps, D. E. Graham, J. W Moon. J. Mater. Chem. C. 2015, 3, 644-650.

22) R. Sankar, R. Maheswari, S. Karthik, K. S. Shivashangari, V. Ravikumar, Mater. Sci. Eng. C. 2014, 44, 234-239.

23) G. Jayakumarai, C. Gokulpriya, R. Sudhapriya, G. Sharmila, C. Muthukumaran, Appl. Nanosci. 2015, 5, 1017-1021.

24)M. A. Ben Aissa, B. Tremblay, A. Andrieux-Ledier, E. Maisonhaute, N. Raouafi, A. Courty, Nanoscale 2015, 7, 3189-3195.

25) S. S. Acharyya, S. Ghosh, R. Tiwari, C. Pendem, T. Sasaki, R. Bal, ACS Catal. 2015, 5, 2850-2858.

26) R. Bal, M. Tada, T. Sasaki, Y. Iwasawa, Angew. Chem. Int. Ed. 2006, 45, 448452.

27) R. J. I. Schmidt, Appl. Catal. A 2005, 280, 89-103.

28) J W. Han, J. Jung, Y.-M. Lee, W. Nam, S. Fukuzumi, Chem. Sci., 2017, 8, 7119.

29) Y. Morimoto, S. Bunno, N. Fujieda, H. Sugimoto, S. Itoh, J. Am. Chem. Soc. 2015, 137, 5867-5870.

30) L. Zhang, S. Qiu, G. Jiang, G. Jiang, R. Tang, Asian J. Org. Chem. 2018, 7,165170. 
31)Z.,Yu, Z. Ma, J. Tang, N. Yan, Y. Du, S. Xi, K. Wang, W. Zhang, H. Wen, J. Wang, Nat. Commun. 2018, 9, 2931.

32) T. Zhang, D. Zhang, X. Han, T. Dong, X. Guo, C. Song, R. Si, W. Liu, Y. Liu, Z. Zhao, J. Am. Chem. Soc. 2018, 140, 16936-16940.

33) Y. Zhu, W. Sun, J. Luo, W. Chen, T. Cao, L. Zheng, J. Dong, J. Zhang, M. Zhang, Y. Han, C. Chen, Q. Peng, D. Wang, Y. Li, Nat. Commun. 2018, 9, 3861.

34) P. Arab, A. Badiei, A. Koolivand, G. Mohammadi-Ziarani, Chinese J. Catal. 2011, 32, 258-263. 